\title{
Marketing mix through the integration of new and traditional media : a review of recent literature.
}

\begin{abstract}
The advertising trend has stimulated towards communicating persuasive messages of a product using two or more media, especially through the integration of traditional media and new media. Contributing to this current issue in advertising research, the purpose of this paper is to explore the current advertising trend through the latest literature on integration of marketing communication of new media and traditional media. The investigation through the traditional medium (television) and the new medium (the internet), which in particular was based on the peripheral routes of persuasion using celebrity endorsement, showed support that the integration of these two mediums can promote memory performance and encourage positive attitudes on the part of consumers. The position of new media especially the internet has also progressed rapidly for the past years. Therefore, new media can play a significant role in contributing to the effectiveness of a marketing mix when integrated with traditional media, specifically with television.
\end{abstract}

Keyword: Marketing communication; Integrated; advertising; Traditional media; New media; Internet; Marketing mix; British consumers. 